\title{
ON THE FEASIBILITY OF AXIAL TRACKING OF A FLUORESCENT NANO-PARTICLE USING A DEFOCUSING MODEL
}

\author{
Nadja Subotic, Dimitri Van De Ville, Michael Unser \\ Biomedical Imaging Group \\ Swiss Federal Institute of Technology Lausanne (EPFL) \\ CH-1015 Lausanne, Switzerland
}

\begin{abstract}
The image of a sub-resolution nano-particle in fluorescence microscopy corresponds to a slice of the 3D point spread function (PSF). This slice relates to the out-of-focus distance of the nano-particle. In this paper, we investigate to which extent it is possible to estimate the out-of-focus distance of the nano-particle from a 2D image based on the knowledge of the 3D PSF.

To this end, we compute the Cramer-Rao bound (CRB) that provides a lower bound on the error of the best estimator of the axial position. The calculation of the CRB involves the specification of a 3D PSF model, the assumption of a signal-dependent Poisson noise, and some acquisition parameters. Our derivation shows that the CRB depends on the defocusing distance. Interestingly, nanometer precision can be attained over a range of defocus distances and for sufficiently high SNR levels. The theoretical results are confirmed with simulated experiments using estimators based on the least-squares (LS) and normalized cross-correlation (NCC) criterion. The results obtained are very close to the theoretical CRB.
\end{abstract}

\section{INTRODUCTION}

Single particle tracking (SPT) has numerous applications in the fields of biophysics [1], cell biology [2, 3], and singlemolecular research studies [4]. Special techniques have been developed to perform precise 3D-SPT [5, 6]. Most notably in [7], it is demonstrated experimentally that precise $3 \mathrm{D}$ tracking of a nano-particle can be done from a $2 \mathrm{D}$ image sequence in video fluorescence microscopy.

A nano-particle with a diameter below the microscope's resolution can be approximated as a point source. Consequently, its 2D image is directly related to the PSF at the corresponding defocusing distance. Speidel et al. [7] looked at a nano-particle that is sufficiently out-of-focus. They experimentally observed a linear relationship between the radius of the outermost ring of the nano-particle's image and its out-of-focus distance. Using this link, they obtained an estimate of the defocusing distance based on the measurement of the radius of the imaged nano-particle.

In this paper, we propose to improve the estimation of the nano-particle's axial position by using all the image information available - not just the outer ring. We investigate the feasibility of this model-based approach and determine its theoretical limits. To this end, we compute the CRB based on a 3D PSF for a typical fluorescence microscopy setting and a Poisson noise model. We examine the CRB as function of the true axial position of the nano-particle for different noise levels and window sizes. We observe a high CRB around the in-focus position, while the best performance is predicted at a defocusing distance of $0.5-1.0$ $\mu \mathrm{m}$.

The theoretical results are validated on simulation experiments using estimators based on the least-squares (LS) and normalized cross-correlation (NCC) criterion. Both methods build a reference stack from the 3D PSF model that is then matched to the data to find the optimal axial distance. Although neither of these methods is the maximumlikelihood estimator (MLE), their precision becomes very close to the one predicted by the CRB.

This paper is organized as follows. In Sect. 2, we describe the image formation model used throughout our work. We give the theoretical 3D PSF, and we formulate a Poisson-noise model that is appropriate for fluorescence microscopy. In Sect. 3, we compute and discuss the CRB. In Sect. 4, LS and NCC estimators are applied for simulated experiments.

\section{IMAGING MODEL}

The image formation in fluorescence microscopy can be described as:

$$
f\left(x, y ; z_{0}\right)=(s * h)\left(x, y, z_{0}\right)
$$

where $*$ denotes the convolution, $s(x, y, z)$ the $3 \mathrm{D}$ object intensity distribution, $h(x, y, z)$ the 3D PSF of the microscope. The resulting $2 \mathrm{D}$ image $f\left(x, y ; z_{0}\right)$, taken at the 
detector plane of the microscope, corresponds to a certain slice at $z=z_{0}$. For a sub-resolution nano-particle, the object intensity distribution is represented by a Dirac; i.e., $s(x, y, z)=\delta(x, y, z)$.

In Fig. 1, the image formation is depicted for two single point sources at different positions on the optical axis. The optical system of the microscope is shown as a single lens. The rays in full line originate from an in-focus point source at $z_{0}=0$ and properly focus on the detector plane. From the other hand, the rays in dotted line originate from an outof-focus point source at $z_{0} \neq 0$ and give rise to a defocused image.

In general, the defocusing effect of the microscope for a point source placed at axial position $z$, is described by the 3D PSF evaluated at the according axial distance. The acquired image corresponds to the sampled, noisy version:

$$
\begin{aligned}
g\left[i, j ; z_{0}\right] & =f\left(i T_{x}, j T_{y} ; z_{0}\right)+n[i, j] \\
& =h\left(i T_{x}, j T_{y}, z_{0}\right)+n[i, j]
\end{aligned}
$$

where $n[i, j]$ represents the signal-dependent noise, and $T_{x}, T_{y}$ are the sampling steps in the $x$ and $y$ directions, respectively.

\subsection{Defocusing model}

The expression of the 3D PSF at the object side in fluorescence microscopy is given by [8]:

$$
\begin{aligned}
h(x, y, z)= & \mid \frac{M}{d_{1}^{2} \lambda^{2}} \int_{0}^{1} R(\rho) \exp \left(\frac{-i 4 \pi \sin ^{2}\left(\frac{\alpha}{2}\right) z \rho^{2}}{\lambda}\right) \\
& \left.J_{0}\left(\rho \frac{2 \pi \sqrt{x^{2}+y^{2}} \sin \alpha}{\lambda}\right) 2 \pi \rho d \rho\right|^{2},
\end{aligned}
$$

where $R(\rho)$ is the pupil function of the lens, $J_{0}$ is a Bessel function of the first kind of order zero, $\alpha$ is a maximal angle of convergence, $M$ is the magnification factor of the lens, $d_{1}$ is the axial working distance from the object to the lens, and $\lambda$ is the emission wavelength. For a circular lens, the pupil function $R(\rho)$ simply reduces to one.

The axial working distance is $d_{1}=d_{10}+z$, where $d_{10}$ is the distance from the in-focus plane to the lens. Since $d_{10}>>z$, we can safely approximate $d_{1} \approx d_{10}$. Thus $\frac{M}{d_{1}^{2} \lambda^{2}}$ can be considered as a constant multiplication factor. The parameters that we use further on are for a typical fluorescence microscope: $\mathrm{NA}=1.25, \lambda=550 \mathrm{~nm}, n_{\mathrm{oil}}=1.515$, $T_{x}, T_{y}=52 \mathrm{~nm}, \alpha=2 \sin ^{-1}\left(\mathrm{NA} / n_{\mathrm{oil}}\right)$.

\subsection{Noise model}

In fluorescence microscopy, the dominant source of the noise originates from the uncertainty in photon counting of

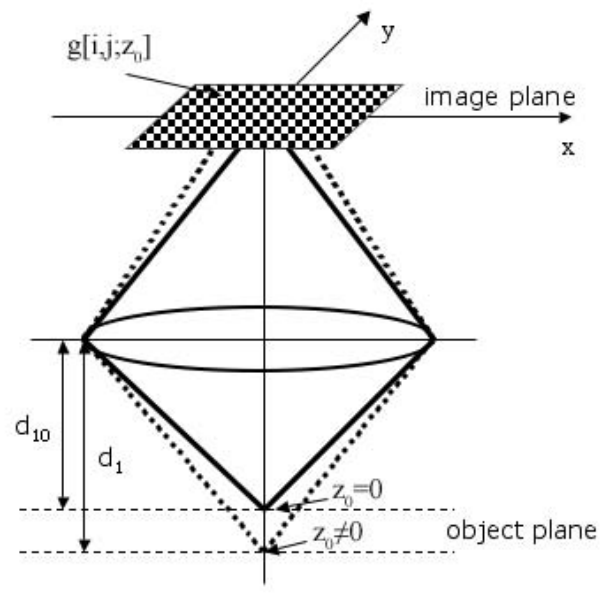

Fig. 1. Image formation of two point sources: the in-focus point source at $z_{0}=0$ and the out-of-focus point source at $z_{0} \neq 0$.

the detector devices, which can be accurately modeled as a Poisson process.

Let $g_{\text {ref }}[i, j ; z]$ be the noise-free intensity level at the detector plane for a pixel $[i, j]$ when the axial position of the nano-particle is $z$. Then, we can write the number of expected photons as:

$$
q_{\mathrm{ref}}[i, j ; z]=c g_{\mathrm{ref}}[i, j ; z]
$$

where $c$ is the conversion factor; i.e., the number of photons needed to increase intensity with one quantization level. The detected number of photons for each pixel follows a Poisson distribution with mean $q_{\mathrm{ref}}[i, j ; z]$ [9].

In general, the $\mathrm{SNR}$ is given by $\mathrm{SNR}=10 \log \left(E_{s} / E_{n}\right)$, where $E_{s}$ and $E_{n}$ denote the signal and noise power, respectively. Since the detection of photons at each pixel is an independent Poisson process, the signal and noise powers are given by

$$
E_{s}=\sum_{i, j} q_{\mathrm{ref}}^{2}[i, j ; z], \quad E_{n}=\sum_{i, j} q_{\mathrm{ref}}[i, j ; z] .
$$

Therefore, the expression of the SNR can be rewritten as

$$
10 \log \frac{\sum_{i, j} q_{\mathrm{ref}}^{2}[i, j ; z]}{\sum_{i, j} q_{\mathrm{ref}}[i, j ; z]}=10 \log \frac{\sum_{i, j} c^{2} g_{\mathrm{ref}}^{2}[i, j ; z]}{\sum_{i, j} c g_{\mathrm{ref}}[i, j ; z]}
$$

To compute the CRB or to simulate experimental data, one typically wants to specify the desired SNR for the infocus image. The corresponding conversion factor is then

$$
c=10^{\frac{\mathrm{SNR}}{10}} \frac{\sum_{i, j} g_{\mathrm{ref}}[i, j ; z]}{\sum_{i, j} g_{\mathrm{ref}}^{2}[i, j ; z]}
$$




\section{CRAMER-RAO BOUND}

The CRB yields the lower bound on the error for any unbiased estimator of a parameter [10]. It requires the determination of the log-likelihood function $\prod_{i, j \in S} \ln P\left(q\left[i, j ; z_{0}\right]\right)$ for the photon counts $q\left[i, j ; z_{o}\right]$ at each observed pixel $[i, j]$ in a support $S$. According to our noise model, $P(\cdot)$ represents the Poisson probability density function. The CRB states that

$$
\operatorname{Var}(\hat{z}) \geq \frac{1}{-E\left[\frac{\partial^{2} \prod_{i, j \in S} \ln P\left(q\left[i, j ; z_{0}\right]\right)}{\partial z^{2}}\right]},
$$

where $\hat{z}$ is our estimate of the true axial position $z_{0}$. In practice, we choose the support $S$ to be a squared template around the imaged nano-particle (see Fig. 3).

Now we can introduce the Poisson probability density function, given by

$$
P\left(q\left[i, j ; z_{0}\right]\right)=\frac{e^{-q_{\mathrm{ref}}[i, j ; z]} q_{\mathrm{ref}}[i, j ; z]^{q\left[i, j ; z_{0}\right]}}{q\left[i, j ; z_{0}\right] !}
$$

where $q_{\text {ref }}[i, j ; z]$ is the noise-free photon count at pixel $[i, j]$ and defocusing distance $z$. The CRB simplifies to

$$
\operatorname{Var}(\hat{z}) \geq 1 / \sum_{i, j \in S} \frac{\left(\frac{\partial q_{\mathrm{ref}}[i, j ; z]}{\partial z}\right)^{2}}{q_{\mathrm{ref}}[i, j ; z]}
$$

with

$$
\begin{aligned}
\frac{\partial q_{\mathrm{ref}}[i, j ; z]}{\partial z}= & \int_{0}^{1} \cos \left(c_{0} z \rho^{2}\right) J_{0}\left(c_{1} \rho \sqrt{x^{2}+y^{2}}\right) 2 \pi \rho d \rho \\
& \cdot \int_{0}^{1} \sin \left(c_{0} z \rho^{2}\right) J_{0}\left(c_{1} \rho \sqrt{x^{2}+y^{2}}\right) \pi \rho^{3} d \rho \\
- & \int_{0}^{1} \sin \left(c_{0} z \rho^{2}\right) J_{0}\left(c_{1} \rho \sqrt{x^{2}+y^{2}}\right) 2 \pi \rho d \rho \\
& \cdot \int_{0}^{1} \cos \left(c_{0} z \rho^{2}\right) J_{0}\left(c_{1} \rho \sqrt{x^{2}+y^{2}}\right) \pi \rho^{3} d \rho,
\end{aligned}
$$

where $c_{0}=4 \pi \sin ^{2}(\alpha / 2) / \lambda$ and $c_{1}=2 \pi \sin \alpha / \lambda$.

We now have all elements to calculate the CRB. In Fig. 2 (a), we show the standard deviation on the axial coordinate estimate as obtained by the CRB. Clearly, the CRB depends on the axial position. Near the in-focus position, the term (10) becomes very small, resulting into a high error estimate. Interestingly, the most accurate estimation (i.e., lowest error bound) is obtained between 0.5 and $1.0 \mu \mathrm{m}$. More defocus increases the error bound, mainly due to the relative decrease of signal. Each curve corresponds to a different SNR in the in-focus plane. In Fig. 2 (b), the CRB is plotted for different template sizes at three axial positions. Depending on the expected axial range to be covered and SNR, these curves can help to adequately select the template size.

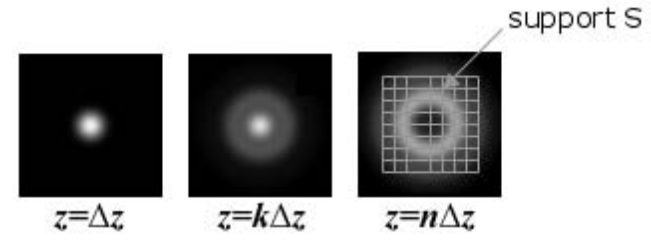

Fig. 3. Reference stack of a nano-particle placed at different axial positions $z=k \Delta z,(k=1,2, . ., n)$.

\section{VALIDATION BY ESTIMATION METHODS}

We describe two axial position estimation algorithms (i.e., based on LS and NCC), and we validate our theoretical findings by performing simulations.

First, we construct a uniformly sampled reference stack of the 3D PSF model; i.e., each slice corresponds to the image of a nano-particle placed at a given axial position, as shown in Fig. 3. The axial spacing $\Delta z$ should be sufficiently small to allow high precision. Next, we simulate experimental data by computing the intensity level according to (2) for a specified axial position. For a given SNR, the conversion factor links the intensity level to the average photon count, which provides the mean of the Poisson process. The noisy simulated image data is obtained as a realization of this process, which is then converted to intensity level values.

In the first estimation method, the axial position is estimated by minimizing the squared error between the measured nano-particle and the reference stack:

$$
\hat{z}=\Delta z \cdot \arg \min _{k} \sum_{i, j \in S}\left(g[i, j ; z]-g_{\text {ref }}[i, j ; k \Delta z]\right)^{2} .
$$

In the second method, we maximize the normalized cross-correlation coefficient between the measured nanoparticle and the reference stack:

$$
\hat{z}=\Delta z \cdot \arg \max _{k} \frac{\sum_{i, j \epsilon S} g[i, j ; z] g_{\mathrm{ref}}[i, j ; k \Delta z]}{\sqrt{\sum_{i, j \epsilon S} g[i, j ; z]^{2}} \sqrt{\sum_{i, j \epsilon S} g_{\mathrm{ref}}[i, j ; k \Delta z]^{2}}} .
$$

We have initially selected the axial spacing of the reference stack below the CRB: $\Delta z=1 \mathrm{~nm}$. In Fig. 4, we show the average error for both estimators using 20 realizations for each axial position. We also mention that the standard deviation on this error varies between 5 and $20 \mathrm{~nm}$. Clearly, the CRB is confirmed by these measurements for each estimator.

From a practical point of view, the LS method is sensitive to the intensity scaling of a reference stack and requires a background subtraction. The NCC, on the other hand, is a shape-based method, which makes it more flexible and a good candidate for a robust implementation. The CRB can be applied to adequately select the spacing between slices in the reference stack. 
(a)

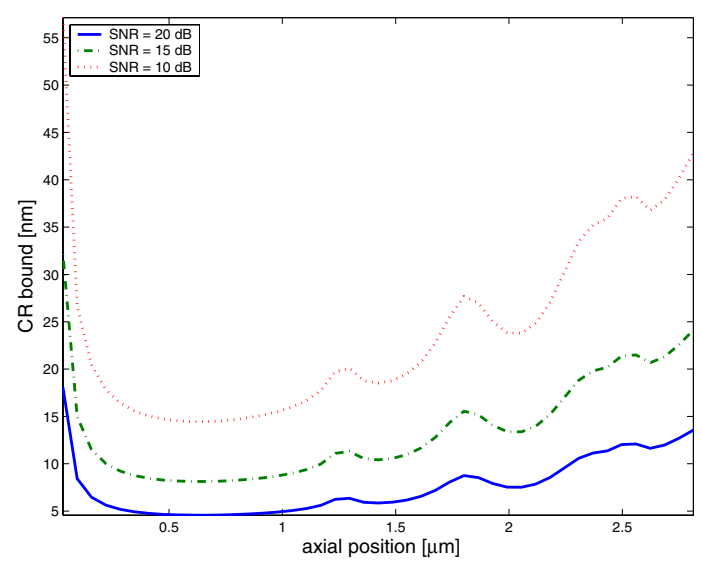

(b)

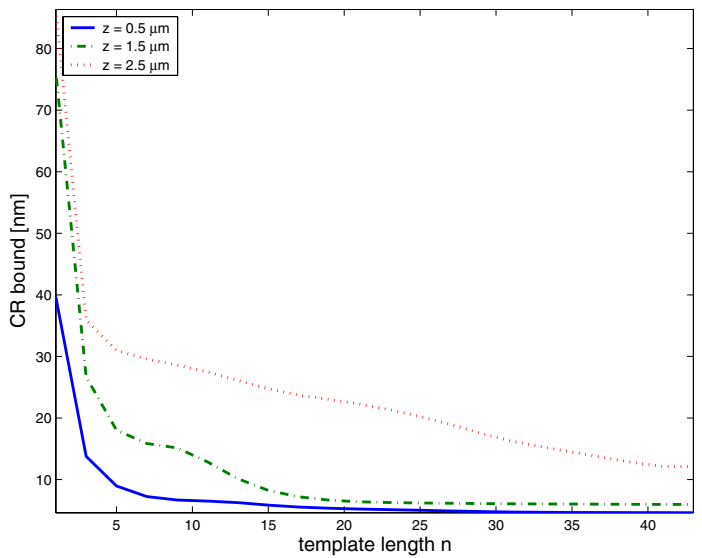

Fig. 2. (a) Influence of the SNR on the CRB for a fixed template size of $43 \times 43$. Notice that the SNR is defined for the in-focus position. (b) Influence of the template size $n \times n$ on the CRB for various defocusing distances. The SNR is fixed at $20 \mathrm{~dB}$.

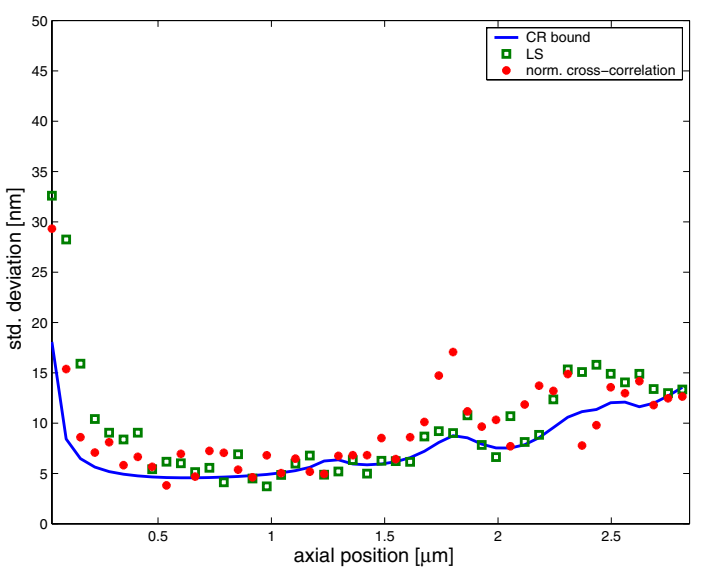

Fig. 4. Average error of the LS and NCC estimation methods (SNR of $20 \mathrm{~dB}$, template size $43 \times 43$ ).

\section{CONCLUSION}

In this paper, we investigated the feasibility of axial tracking of a nano-particle. The image formation model incorporates a 3D PSF (for the defocusing effect) and a Poisson noise model. We computed the theoretical lower bound for the error in the estimation of the axial position. We performed simulation experiments which confirm our theoretical findings.

In the future work, we intend to further validate our results using real experimental data. We will also extend the technique for tracking moving nano-particles.

\section{REFERENCES}

[1] H. Qian, M. Sheetz, and E. Elson, "Single particle tracking: Analysis of diffusion and flow in two-dimensional systems," Biophys J., vol. 60(4), pp. 910-21, 1991.

[2] M. J. Saxton and K. Jacobson, "Single-particle tracking: Applications to membrane dynamics," Annu. Rev. Biomol. Struct., vol. 26, pp. 373-99, 1997.

[3] Y. Sako, A. Nagafuchi, S. Tsukita, M. Takeich, and A. Kusumi, "Cytoplasmic regulation of the movement of Ecadherin on the free cell surface as studied by optical tweezers and single particle tracking: Corralling and tethering by the membrane skeleton," J. Cell Biol., vol. 140, no. 5, 1998.

[4] U. Kubitscheck, "Single protein molecules visualized and tracked in the interior of eukaryotic cells," Single Mol., vol. 3, no. 5-6, pp. 267-274, 2002.

[5] P. T. So, T. Ragan, E. Gratton, J. Carerro, and E. Voss, "Twophoton single particle tracking in 3d," SPIE Proceedings, vol. 2983, pp. 45-56, 1997.

[6] A. Pralle, M. Prummer, E. L. Florin, E. H. K. Stelzer, and J. K. H. Horber, "Three-dimensional high-reoslution particle tracking for optical tweezers by forward scattered light," Microscopy research and technique, vol. 44, pp. 378-386, 1999.

[7] M. Speidel, A. Jonas, and E. Florin, "Three-dimensional tracking of fluorescent nanoparticles with subnanometar precision by use of off-focus imaging," Optics Letters, vol. 28, no. 2, Jan. 2003.

[8] M. Gu, Advanced Optical Imaging Theory, Springer Series in Optical Sciences, 1999.

[9] G. Kempen, "Image restoration in fluorescence microscopy," PhD Thesis, 1999.

[10] H. Cramer, Mathematical Methods of Statistics, Princeton, NJ: Princeton University Press., 1946. 